\title{
Acknowledgement to Reviewers of Antibiotics in 2018
}

\author{
Antibiotics Editorial Office \\ MDPI, St. Alban-Anlage 66, 4052 Basel, Switzerland \\ Published: 10 January 2019
}

Rigorous peer-review is the corner-stone of high-quality academic publishing. The editorial team greatly appreciates the reviewers who contributed their knowledge and expertise to the journal's editorial process over the past 12 months. In 2018, a total of 108 papers were published in the journal, with a median time to first decision of 15.5 days and a median time to publication of 37 days. The editors would like to express their sincere gratitude to the following reviewers for their cooperation and dedication in 2018:

Aabenhus, Rune

Abboud, Martine

Abejón, Ricardo

Aldred, Katie

Andrianasolo, Eric

Arango, Rachel A.

Armstrong, Glen

Arndt, Patrick G.

Asamizu, Shumpei

Ashiru-Oredope, Diane

Ashish, Pathak

Avent, Minyon

Bach, Horacio

Baerga-Ortiz, Abel

Baliraine, Frederick

Bardi, Giuseppe

Beld, Joris

Berkes, Charlotte

Bernardini, Giulia

Bøgwald, Jarl

Briani, Federica

Brown, Stanley

Bugla-Płoskońska, Gabriela

Caffrey, Patrick

Cain, Ricky

Campos Da Rosa, Joaquim M.

Chamakura, Karthik

Charvat, Robert A.

Chattoraj, Dhruba

Chen, Long

Chiarelli, Laurent

Chodosh, James

Chopra, Teena
Choudhary, Kumari Sonal

Coleman, Anthony

Constance, Jonathan E.

Córdoba, Gloria

Criss, Alison K.

Crossley, Alison

Crumlish, Mags

Cryle, Max

Currie, Kay

Dallinger, Reinhard

D'Andrea, Marco Maria

Danziger, Larry $\mathrm{H}$.

David, Michael Z.

De Peralta, Tracy L.

De Piccoli, Giacomo

Dewulf, Jeroen

Díaz Martínez, Margarita

Dixon, Nicholas

Dkhar, Hedwin Kitdorlang

Domingo-Calap, Pilar

Drlica, Karl

Eckroat, Todd

Edwards, Adrianne N.

El Badawy, Amro

Else, Terry Ann

El-Seedi, Hesham R.

Esteve-Romero, Josep

Eustaquio, Alessandra S.

Fernández, Lucía

Fernandez-Martinez, Lorena

Flemetakis, Emmanouil

Fleming-Dutra, Katherine E.

Floresta, Giuseppe 
Fouladkhah, Aliyar

Franco, Carlos M.

Furneri, Pio Maria

Gao, Zhe

Garcia, Coralia V.

Ghosh, Santosh

Giacobbe, Daniele Roberto

Gile, Gillian

Goh, Shan

Gökce, Bilal

Gomes, Maria S.

Goyer, Claudia

Hadjikakou, Sotiris

Han, Qifeng

Hansford, Karl

Hardies, Stephen C.

Hashimoto, Masaru

Haslinger, Kristina

Hassan, Amany

Hayes, Sidney J.

Hearn, Michael J.

Hendrickson, Heather

Hernalsteens, Jean-Pierre

Hindra, Hindra

Hirakawa, Hidetada

Holmes, Neil A.

Huang, Cheng-Liang

Ito, Takuya

Jain, Prashant

Jankauskaite, Virginija

Jay-Russell, Michele

Johnson, Paul

Jones, Marjorie

Jordao, Luisa

Ju, Kou-San

Kahler, Charlene

Kakkar, Ashok

Kako, Tetsuya

Kalatzis, Panos G.

Kappell, Anthony

Keck, James

Kiljunen, Saija

Kinaciyan, Tamar

Kobayashi, Hideki

Komaki, Hisayuki

Kondo, Tatsuya

Konstantinidis, Theocharis G.

Koutelidakis, Antonios E.

Koyama, Yu

$\mathrm{Ku}$, Seockmo

Kuper, Kristi

Kvítek, Libor
Laanto, Elina

Larabee, Jason L.

Lecky, Donna M.

Leung, Chung Yin (Joey)

Lewis, David

Lognay, Georges

Lohman, Jeremy

Louie, Stacey

Lous, Jørgen

Luedtke, Brandon

Luhavaya, Hanna

Manjunath, Manubolu

Marcone, Giorgia Letizia

Marques, Claudia

Martino, Sabata

Mathieu, Jacques

Matsuda, Yudai

Mendes, Marta V.

Mesquita, João

Metsä-Ketelä, Mikko

Millard, Andrew D.

Minami, Masaaki

Miotla, Pawel

Moll, Gert N.

Motwani, Tina

Murray, Thomas Scot

Nanjappa, Deepak

Nieto, Teresa Pérez

Nimmagadda, Alekhya

Nori, Priya

Novick, Richard P.

Nyerges, Gyorgyi

Ogawara, Hiroshi

Okafor, Florence

Olmos, Dania

Oppong, Raymond

Pajunen, Maria

Papazafiropoulou, Athanasia

Papp, John

Park, Se Chang

Paskaleva, Elena

Pedulla, Marisa

Peng, Teng

Pettus, Thomas

Phuoc, Nghia Truong

Piano, Martina

Pinto, Tatiana

Pomposelli, James

Puiggalí, Jordi

Pyatenko, Alexander

Ragusa, Andrea

Rahme, Kamil 
Raposio, Edoardo

Reid, Christopher W.

Roh, Changhyun

Roque, Fátima

Sabatier, Jean-Marc

Sacco, Keith A.

Saiz Jimenez, Cesareo

de la Salud-Bea, Roberto

Santander, Javier

Schaaper, Roel

Schäberle, Till F.

Schmidt, Martin

Schwan, William

Sclavi, Bianca

Scrano, Laura

Seekatz, Anna Maria

Seipke, Ryan

Seo, Keun-Seok

Setzer, William N.

Sharkey, Liam

Sheikh, Alaullah

Shen, Yang

Silvestre, Samuel

Sim, Edith

Skandalis, Nikolaos

Smani, Younes

Snyder, Lori

Sorensen, John

Sorg, Olivier

Stein, Daniel

Straight, Paul

Streubel, Robert

Summers, James Kevin

Sutton, Scott

Szewczyk, Katarzyna

Taheri, Shima

Tailhades, Julien

Takahashi, Satoshi
Tamulevičienė, Asta

Tavío, María M.

Tayebati, Seyed Khosrow

Toiu, Anca

Tonkin-Crine, Sarah

Torres-Barceló, Clara

Toscani, Anita

Tyndall, Joel

Ueda, Kenji

Valencia, Gregorio

Valera, Enrique

Vamanu, Emanuel

Van Boeckel, Thomas

Van Lanen, Steven

Vasile, Francesca

Vemula, Harika

Venturini, Carola

Viale, Pierluigi

Vijgenboom, Erik

Wagemans, Jeroen

Wanat, Marta

Wang, Liang-Chun

Weber, J. Mark

Weber, Tilmann

Wendling, Carolin C.

White, Kimberly N.

Wink, Joachim

Witte, Angela

Wu, Changsheng

Xie, Ran

Yang, Lei

Yim, Grace

Yu, Aimin

Zabetakis, Ioannis

Zhang, Fan

Zhu, Jingen

Ziemert, Nadine

(C) 2019 by the authors. Licensee MDPI, Basel, Switzerland. This article is an open access article distributed under the terms and conditions of the Creative Commons Attribution (CC BY) license (http://creativecommons.org/licenses/by/4.0/). 